\title{
Attosecond Interference Induced by Coulomb-Field-Driven Transverse Backward-Scattering Electron Wave-Packets
}

\author{
Xiaohong Song, Peng Liu, Cheng Lin, Zhihao Sheng, Xianhuan Yu, and Weifeng Yang* \\ Department of Physics, College of Science, Shantou University, Shantou, Guangdong 515063, China \\ Shilin $\mathrm{Hu}$ and Jing Chen ${ }^{\dagger}$ \\ HEDPS, Center for Applied Physics and Technology, \\ Collaborative Innovation Center of IFSA, Peking University, Beijing 100084, China and \\ Institute of Applied Physics and Computational Mathematics, P. O. Box 8009, Beijing 100088, China \\ SongPo Xu, YongJu Chen, Wei Quan, and XiaoJun Liv \\ State Key Laboratory of Magnetic Resonance and Atomic and Molecular Physics, \\ Wuhan Institute of Physics and Mathematics, Chinese Academy of Sciences, Wuhan 430071, China
}

(Dated: May 18, 2018)

\begin{abstract}
A novel and universal interference structure is found in the photoelectron momentum distribution of atoms in intense infrared laser field. Theoretical analysis shows that this structure can be attributed to a new form of Coulomb-field-driven backward-scattering of photoelectrons in the direction perpendicular to the laser field, in contrast to the conventional rescattering along the laser polarization direction. This transverse backward-scattering process is closely related to a family of photoelectrons initially ionized within a time interval of less than 200 attosecond around the crest of the laser electric field. Those electrons, acquiring near-zero return energy in the laser field, will be pulled back solely by the ionic Coulomb field and backscattered in the transverse direction. Moreover, this rescattering process mainly occurs at the first or the second return times, giving rise to different phases of the photoelectrons. The interference between these photoelectrons leads to unique curved interference fringes which are observable for most current intense field experiments, opening a new way to record the electron dynamics in atoms and molecules on a time scale much shorter than an optical cycle.
\end{abstract}

PACS numbers: 32.80.Wr, 33.60.+q, 61.05.jp

In the ionization process of atoms in intense laser field, the electron wave packet (EWP) may follow different paths from its bound state to the continuum in the combined laser and Coulomb fields. The interference between the EWPs might create richly structured patterns in the final photoelectron distribution, which inherently encode the temporal and spatial information of the ions and electrons. For example, a holographic interference structure was recently observed in the photoelectron momentum distribution (PMD) of metastable xenon atom ionized by a $7 \mu \mathrm{m}$ free-electron laser pulse [1]. This interference structure was explained as interference between the direct and the laser-driven forward-scattered EWPs generated within the same quarter-cycle of the laser pulse, providing an efficient way in exploring the structure and the dynamics of the atoms and molecules with attosecond temporal and angstrom spatial resolution. Thereafter, the photoelectron interference structure has been extensively investigated for a broad range of laser parameters covering tunneling to multiphoton ionization regimes, however, a full understanding of its underlying physics has not yet been achieved [2 8 ]

Note that for the formation of this specific holographic interference structure, the reference wave, i.e., the direct electron, upon ionization, was assumed to be very weakly affected by the ionic Coulomb field, while the rescattered one, experienced strong Coulomb focusing and passed close to the ion, was considered as the signal wave. Moreover, previous studies have suggested that the Coulomb field plays a negligible role in the holographic interference patterns 1, 2, 4]. On the other hand, it has been generally accepted that the ionic Coulomb field plays a pivotal role in the photoelectron dynamics, e.g., giving rise to an unexpected "low-energy" structure in the photoelectron energy spectrum [9 16] and a clear minimum at zero in the electron momentum distribution along the laser polarization direction [17, 18]. An open question thus concerns if the ionic Coulomb field would find its fingerprints in more general interference patterns, if not in the specific holographic interference, and more importantly, under which circumstance and to which extent the ionic Coulomb field would play a role in the interference pattern.

In this letter, we show that the ionic Coulomb potential can leave a significant imprint on the interference structure in the photoelectron momentum spectrum of atoms, which is demonstrated experimentally by a novel and universal curved interference pattern in the PMD. Using a recently developed generalized quantum-trajectory Monte Carlo (GQTMC) method, we clarify that this new structure can be attributed to a Coulomb-field-driven transverse backward-scattering process. In contrast to 
conventional rescattering which happens in the laser polarization direction, when electrons emitted around the peaks of the laser electric field come back to the core with near-zero drift energy in the laser polarization direction, they will be pulled back solely by the Coulomb potential and backward scattered upon the core in the direction of perpendicular to laser polarization axis. The interference among the Coulomb-field-driven transverse backward-scattering electrons, initially emitted near the crest of the oscillating electric field, can induce a distinct interference structure, which can be well distinguished from other interference structures, e.g., the welldocumented holographic interference structure.

The experiments have been performed with cold target recoil-ion momentum spectroscopy (COLTRIMS) $[19,20]$. The laser pulse was generated by a commercial Ti: Sapphire femtosecond laser system (FEMTOPOWER, Femtolasers Produktions $\mathrm{GmbH}$ ) with a center wavelength of $800 \mathrm{~nm}$, a pulse duration of $30 \mathrm{fs}$ and a repetition rate of $5 \mathrm{kHz}$. The pulse energy was controlled by means of an achromatic half wave plate followed by a polarizer. The laser beam was directed and focused into a supersonic Ar gas jet inside the COLTRIMS vacuum chamber. The photoelectrons and $\mathrm{Ar}^{+}$ions created in the interaction region were accelerated by a uniform weak electric field $(3.8 \mathrm{~V} / \mathrm{cm})$ towards two positionsensitive Microchannel plate (MCP) detectors. A pair of Helmholtz coils generated a weak uniform magnetic field (7.8 Gauss) to confine the electron movement perpendicular to the electric field. The three dimensional vector momenta of photoelectrons and $\mathrm{Ar}^{+}$were obtained from their times of flight and the impact positions.
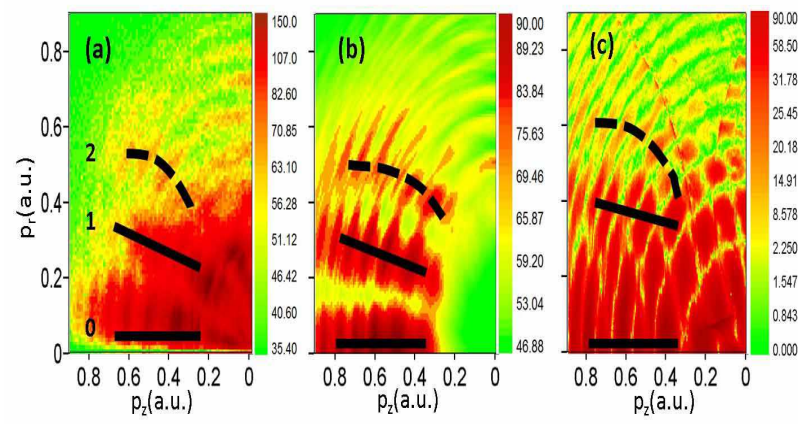

FIG. 1: (Color online) Experimental and simulated twodimensional photoelectron momentum spectra of Ar atom. (a) Experimental result; (b) Focal-averaged TDSE simulation; (c) GQTMC simulation. Laser intensity $I=1.7 \times 10^{14}$ $\mathrm{W} / \mathrm{cm}^{2}$, the wavelength $\lambda=800 \mathrm{~nm}$, and the pulse duration is $30 \mathrm{fs}$.

Fig. 1(a) shows the experimental PMD from Ar atom driven by an $800 \mathrm{~nm}$ laser pulse with intensity of $1.7 \times 10^{14}$ $\mathrm{W} / \mathrm{cm}^{2}$. The laser pulse is linearly polarized along the $\mathrm{z}$ axis. Holographic interference stripes (marked by solid line) can be clearly seen [1]. Moreover, an additional interference fringes (marked by dash line) can also be observed in Fig. 1(a). Unlike the holographic interference fringes which are almost straight [8], this interference fringe clearly shows an arc shape. Calculation using the time-dependent Schrödinger equation (TDSE) [6] well reproduces the experimental observation including both the conventional holographic and curved stripes (see Fig. 1(b)). In the simulation, focal-averaging over the laser intensities in the focus is considered.

To reveal the underlying mechanism of this new interference structure, we apply a generalized quantumtrajectory Monte Carlo method to calculate the PMD which is depicted in Fig. 1(c). The GQTMC method is based on the nonadiabatic ionization theory 21, 22], classical dynamics with combined laser and Coulomb fields 23 25], and the Feynman's path integral approach [26, 27]. Briefly, the evolution of the EWPs in the laser field is simulated by launching randomly a set of electron trajectories with different initial conditions. The weight of each electron trajectory is given by $w\left(\mathbf{v}_{r 0}, t_{0}\right)=$ $\Gamma\left(t_{0}\right) \Omega\left(\mathbf{v}_{r 0}, t_{0}\right)$. Here $\Gamma\left(t_{0}\right)$ is the ionization rate. Different from the conventional QTMC model [27] where the Ammosov-Delone-Krainov (ADK) ionization rate is used, our GQTMC method is based on the nonadiabatic ionization theory [21, 22] so that it can be applied to a quite large Keldysh parameter range. The details and the comparison among the TDSE, QTMC and GQTMC methods will be described elsewhere [28]. The instantaneous ionization rate is given as 21, 22]

$$
\Gamma(t)=N(t) \exp \left(-\frac{E_{0}^{2} f^{2}(t)}{\omega^{3}} \Phi(\gamma(t), \theta(t))\right) .
$$

Here, $E_{0} f(t)$ and $\theta(t)$ are the envelope and the phase of the laser electric field, respectively. The pre-exponential factor is

$$
\begin{gathered}
N(t)=A_{n^{*}, l^{*}} B_{l,|m|}\left(\frac{3 \kappa}{\gamma^{3}}\right)^{\frac{1}{2}} C I_{p}\left(\frac{2\left(2 I_{p}\right)^{3 / 2}}{E(t)}\right)^{2 n^{*}-|m|-1} \\
\kappa=\ln \left(\gamma+\sqrt{\gamma^{2}+1}\right)-\frac{\gamma}{\sqrt{\gamma^{2}+1}}
\end{gathered}
$$

where $I_{p}$ the ionization potential, and the coefficient $A_{n^{*}, l^{*}}$ and $B_{l,|m|}$ coming from the radial and angular part of the wave function are given by Eq.(2) in Ref. [21]. $\quad C=\left(1+\gamma^{2}\right)^{|m| / 2+3 / 4} A_{m}(\omega, \gamma)$ is the Perelomov-Popov-Terent'ev correction to the quasistatic limit $\gamma \ll 1$ of the Coulomb pre-exponential factor with $A_{m}$ given by Eqs. (55) and (56) in Ref. [22]. $\Omega\left(\mathbf{v}_{r 0}, t_{0}\right)=\left[\mathbf{v}_{r 0} \sqrt{2 I_{p}} /\left|E\left(t_{0}\right)\right|\right] \exp \left[\sqrt{2 I_{p}}\left(\mathbf{v}_{r 0}\right)^{2} /\left|E\left(t_{0}\right)\right|\right]$ is the electrons' initial transverse velocity distributions. For consistence, the coordinate of the tunnel exit is also modified to be as $z_{0}=\frac{2 I_{p}}{E\left(t_{0}\right)}\left(1+\sqrt{1+\gamma^{2}\left(t_{0}\right)}\right)^{-1}[22]$. After ionization, the classical motion of the electrons in the combined laser and Coulomb fields is governed by the Newtonian equations. The phase of the $j$ th electron 
trajectory can be expressed as 26,27 .

$$
S_{j}\left(p, t_{0}\right)=\int_{t_{0}}^{\infty}\left\{\left(\frac{v_{p}^{2}(\tau)}{2}+I_{p}-\frac{Z_{\text {eff }}}{|r(\tau)|}\right)\right\} d \tau,
$$

where $p$ is the asymptotic momentum of the electron, $Z_{e f f}=\sqrt{2 I_{p}}$ is the effective charge of the ion potential. The EWPs will interfere with each other when they have the same asymptotic momenta. Using a parallel algorithm, one billion electron trajectories were calculated and added coherently to obtain the PMD. The probability of each asymptotic momentum is determined by

$$
\left|\Psi_{p}\right|^{2}=\left|\sum_{k} \sqrt{\Gamma\left(t_{0}, v^{k}\right)} \exp \left(-i S_{k}\right)\right|^{2} .
$$

As shown in Fig. 1(c), the main features of the interference fringes observed in Figs. 1(a) and 1(b) can be well reproduced by the GQTMC simulation. To gain more insight into the origin of the curved interference structure, we analyze all electron trajectories contributing to the momentum spectrum with final longitudinal momentum in the range 0.3a.u. $\leq \mathbf{p}_{z}$. In this region, both holographic fringes and curved interference fringes can be clearly seen. Fig. 2(a) shows the distribution of the initial tunneling phase and initial transverse momentum of the trajectories contributing to this momentum range. It can be seen that, for the momentum ranges we are analyzing, the initial conditions of electrons within one laser cycle are separated into four areas (denoted as area A-D in Fig. 2(a)). Figs. 2(b) and 2(d) show the typical electron trajectories from areas B and A, respectively. Obviously, these two areas correspond to different families of the electron trajectories.

There are two types of electron trajectories in area B (see Fig. 2(b)). Electrons with small initial transverse momenta in this area are forward scattered by the ionic potential in the direction of the laser polarization (black lines). While the electrons with large initial transverse momenta only revisit and pass by the core at large distances without scattering, which are considered as the direct electrons (red lines). To identify their contributions to the total momentum spectrum, we then reconstruct the final momentum distribution of electron trajectories only in area B, as shown in Fig. 2 (c). In good agreement with pervious strong-field approximation [1] and classical calculations [8], the interference between these two kinds of EWPs from area B yields the holographic interference structures which is straight and radial. It reproduces the 0th and 1st fringes in the total photoelectron spectrum (see Fig. 1(a)). Obviously, the electron trajectories that are initially launched within area B do not lead to the curved interference pattern.

In Fig. 2 (d), it can be found that electron trajectories initially launched in area A are quite different from those in area B. As more clearly shown in Fig. 2(f), when the electron comes back to around $\mathrm{z}=0$, (i) it has near-zero
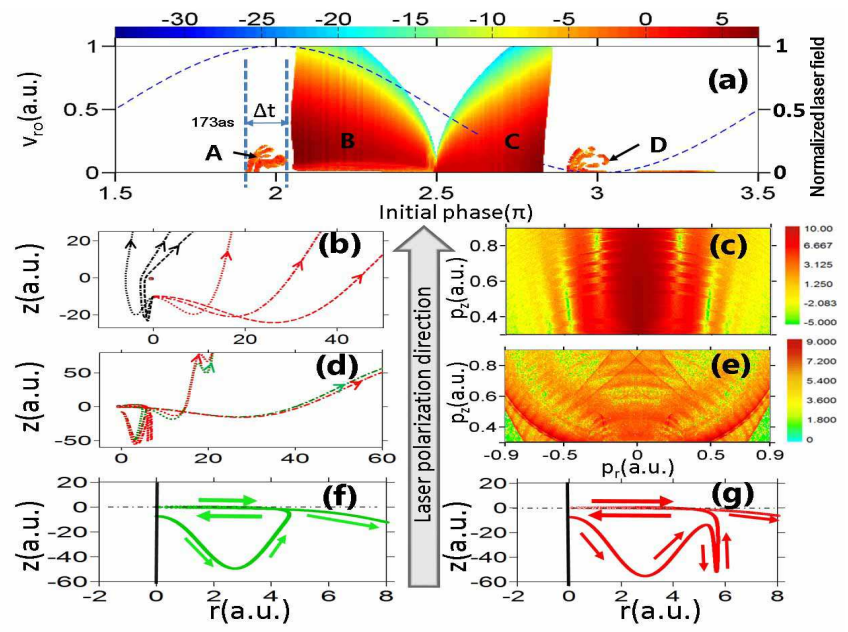

FIG. 2: (Color online) (a) Distributions of the initial transverse velocities and the initial ionization phases for $0.3 a . u . \leq$ $\mathbf{p}_{z}$ in Fig. 1(c). The color code denotes the weights of the electrons in area A-D. (b) typical trajectories of electrons in area B. (c) Reconstructed final momentum distributions of electrons in area B. (d) typical trajectories of electrons in area A. (e) Reconstructed final momentum distributions of electrons in area A. A zoom in of electron trajectories scattered at the first and second return in (d) is shown in (f) and (g), respectively.

returning velocity along the laser polarization, so it almost stops at around $\mathrm{z}=0$ in the laser polarization i.e., $v_{z} \approx 0$. (ii) it is the Coulomb field that pulls the electron back along the line $z=0$ to the core and further induces backward scattering in the direction perpendicular to the laser polarization axis (also along the line $z=0$ ). These two key points make this type of electron trajectories quite special and distinguished in essence from previous widely accepted rescattering process in which the electrons are driven back mainly by the laser field and collide in the laser polarization direction [29]. In this Letter, we refer to this special rescattering as "Coulombfield-driven transverse backward-scattering". Moreover, this Coulomb-field-driven transverse backward-scattering may occur not only at the first time (see Fig. 2(f)) but also at the second time (see Fig. 2(g)) when the electron returns to the core [30]. After scattering upon the core, the electrons may possess the same momentum but apparent different phases, giving rise to the interference fringes observed in Fig. 2(e). It is worthwhile mentioning that the effect of the multiple return trajectories in the interference structure in the PMD has been reported in Ref. 2]. However, the structure discussed there locates in small momentum region and, similar to that proposed in Ref. 1], can be attributed to the interference between undistorted and forward scattered electrons.

Obviously, the Coulomb potential plays a dominant role in the evolution of this kind of electron trajectories. Without the Coulomb potential, these electrons will 
only contribute to the momentum map around $\mathbf{p}_{z} \sim 0$. Recently, it has been reported that the interference between such direct ionization electrons emitted at every electric field's extreme, which are spaced by $T / 2$, will result in a $2 \hbar \omega$ separation of the ATI rings for the perpendicular emission [31]. However, once such electrons are driven back by the Coulomb potential and further backward scattered in the transverse direction, they may have large final momenta both in the longitudinal and transverse directions.

Fig. 2(e) shows the reconstructed final momentum distribution of electrons solely in area A. Most interestingly, the interference among the electrons emitted from one single electric field extremum but experienced Coulombfield-driven transverse backward-scattering at different return times can induce a novel interference structure. These fringes show an arc pattern different from the holographic interference fringes induced by electron trajectories in area B (see Fig. 2(c)) [32]. Clearly, it gives rise to the curved 2nd fringes in both the experimental and theoretical results in Fig. 1. Moreover, some electrons in the area A come out with small final transverse momenta after scattering with the core (dash lines in Fig. $2(\mathrm{~d})$ ) and also form a central interference fringe in Fig. 2(e). This fringe coincides with the 0th fringe formed by the electrons from area B (see Fig. 2(c)). It cannot be distinguished in the total PMD (e. g., see Fig. 1(c)) since the 0 th fringe from area $\mathrm{B}$ in this region dominates. It should be mentioned here that electrons from area $\mathrm{C}$ and $\mathrm{D}$ only contribute to the background of the fringes.

It is worthwhile mentioning that the holographic interference studied in Ref. 1] arises from the interference between the EWPs emitted during the same quarter cycle of the laser field which can be applied to image the sub-cycle dynamics of the photoelectron. Whereas the newly identified curved interference structure is induced by the Coulomb-field-driven transverse backward scattered EWPs that are generated within a time window of only about 0.06 laser cycle $(\sim 173$ as for the $800 \mathrm{~nm}$ laser field used here) around the peak of the laser field. This implies that this novel interference structure can record electron dynamics on a much shorter time scale.

In the total momentum distribution map, these two interference structures will coexist and compete with each other. According to our analysis, the Coulomb potential will become an overwhelming factor for photoelectrons with small longitudinal velocity when they come back to the core, leading to this kind of curved interference fringe. Therefore, the visibility of this structure in the total momentum distribution is dependent on the proportion of such photoelectrons that can be strongly affected by the Coulomb potential. As an estimation, we assume that the return kinetic energies of such photoelectrons are less than the Coulomb potential energy at the tunneling exit. Then the interval of the initial phase around the crest of the laser field for which the above assumption is valid can be given by 33

$$
\delta \phi \propto \frac{\omega^{2}}{E_{0} I_{p}} \propto \frac{1}{U_{p} z_{0}} .
$$

Here $U_{p}=E_{0}^{2} / 4 \omega^{2}$ is the ponderomotive energy. According to Eq. (5), relatively lower intensity and shorter wavelength are favored by the curved interference fringe which is caused by the Coulomb-field-driven transverse backward-scattering process.

In fact, this kind of curved interference structure can be clearly seen in previous experiments of different atoms [5, 31, 34 36]. It can be found that the curved interference structure in these experiments, for examples, Fig. 2 in Ref. 35] and Fig.21(c) in Ref. 34, resembles very well the structure shown in Fig. 2(e), demonstrating that this curved interference pattern is a "universal" structure for different atoms in the typical conditions for most current intense field experiments.

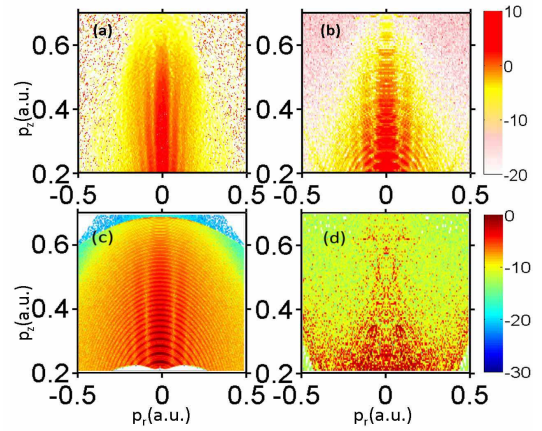

FIG. 3: (Color online) (a) Experimental two-dimensional photoelectron momentum spectra of Xe atom in Ref.[1]; (b) the corresponding GQTMC simulation; (c) the reconstructed final momentum distributions of electrons in area B. (d) the reconstructed final momentum distributions of electrons in area A.

In contrast, this curved interference pattern is invisible in the momentum distribution of Ref. [1] where a $7-\mu \mathrm{m}$ mid-infrared pulse is used. In this circumstance, though the ponderomotive energy is relatively small $\left(U_{p}=0.118\right.$ a.u.), the large tunneling exit $\left(z_{0} \approx 31\right.$ a.u.) makes the influence of the Coulomb potential very weak. As a result, the probability of the Coulomb-field-driven transverse backward-scattering is negligible comparing with the contribution from area B in Fig. 2(a). Figs. 3(a) and 3(b) show the experimental result in Ref. [1] and the corresponding GQTMC simulation, respectively. Again good agreement is achieved between the theoretical and experimental results. Both of them show straight holographic interference fringes and the curved interference fringes are absent. Figs. 3(c) and 3(d) present the reconstructed final momentum distributions of electrons in area B and area A under the same experimental conditions. It can be found that Fig. 3(c) is almost identical to Fig. 3(b), which means that electrons only from 
area $\mathrm{B}$ can well reproduce the main experimental and theoretical PMDs while the electrons from area A play a negligible role in the total PMD. Moreover, in contrast to Fig. 2(e), the interference fringes induced by the Coulomb-field-driven transverse backward-scattering is almost invisible in Fig. 3(d). This is due to that, in this situation, the probability of transverse backward scattering is so low that there are still not enough trajectories in our simulation $\left(1 \times 10^{9}\right.$ total trajectories are used $)$ to make the interference fringes visible in Fig. 3(d).

In summary, a novel curved interference structure is identified in the experimental and theoretical PMDs of atoms in intense infrared laser field. A GQTMC method is able to well reproduce the experimental observation and enable further analysis of the underlying mechanism of this peculiar structure. We demonstrated that, different from the well-documented holographic interference fringes which is attributed to interference between EWPs generated during a quarter cycle of the laser pulse, the curved interference structure originates from the interference among EWPs emitted within an attosecondtimescale window around the crests of the laser field. When these electrons are driven back by the laser field to the core with near-zero longitudinal momenta, they may be pulled back by the ionic Coulomb potential and be further backward scattered in the direction perpendicular to the polarization direction. This scattering may happen at different return times, leading to different phases of the ejected photoelectrons. The interference between these electrons results in obvious curved fringes in the PMD, which can be easily distinguished from the straight radial holographic interference fringes. Analysis shows that this interference structure can be observed for different atoms under the typical conditions of current intense field experiments. Moreover, this interference pattern can be applied to record electron dynamics on a time scale of about $100 \sim 200$ as.

We are grateful to M. Vrakking and Y. Huismans for providing us their experimental data. The work was supported by the National Basic Research Program of China (Grant No. 2013CB922201), the NNSF of China (Grant Nos. 11374202, 11274220, 11274050, 11334009 and 11425414), Guangdong Natural Science Foundation (Grant No. 2014A030311019), and the Open Fund of the State Key Laboratory of High Field Laser Physics (SIOM). W. Y. acknowledges support by the "YangFan" Talent Project of Guangdong Province.

* Electronic address: wfyang@stu.edu.cn

† Electronic address: chen jing@iapcm.ac.cn

\# Electronic address: xjliu@wipm.ac.cn

[1] Y. Huismans et al., Science 331, 61 (2011).

[2] D. D. Hickstein et al., Phys. Rev. Lett. 109, 073004 (2012).
[3] M. Meckel, A. Staudte, S. Patchkovskii, D. M. Villeneuve, P. B. Corkum, and R. Dörner, Nature Phys. 10, 594 (2014).

[4] Y. Huismans et al., Phys. Rev. Lett. 109, 013002 (2012).

[5] T. Marchenko, Y. Huismans, K. J. Schafer, and M. J. J. Vrakking, Phys. Rev. A 84, 053427 (2011).

[6] W. Yang, Z. Sheng, X. Feng, M. Wu, Z. Chen, and X. Song, Opt. Express 22, 2519 (2014).

[7] X. B. Bian, and A. D. Bandrauk, Phys. Rev. Lett. 108, 263003 (2012).

[8] X. B. Bian, Y. Huismans, O. Smirnova, K. J. Yuan, M. J. J. Vrakking, and A. D. Bandrauk, Phys. Rev. A 84, 043420 (2011).

[9] C. I. Blaga et al., Nat. Phys. 5, 335 (2009).

[10] W. Quan et al., Phys. Rev. Lett. 103, 093001 (2009).

[11] C. Wu et al., Phys. Rev. Lett. 109, 043001 (2012).

[12] C. Liu and K. Z. Hatsagortsyan, Phys. Rev. Lett. 105, 113003 (2010).

[13] T. M. Yan, S. V. Popruzhenko, M. J. J. Vrakking, and D. Bauer, Phys. Rev. Lett. 105, 253002 (2010).

[14] A. Kästner, U. Saalmann, and J. M. Rost, Phys. Rev. Lett. 108, 033201 (2012).

[15] L. Guo et al., Phys. Rev. Lett. 110, 013001 (2013).

[16] W. Becker, and D. B. Milošević, J. Phys. B 48, 151001 (2015).

[17] J. Chen and C.H. Nam, Phys. Rev. A 66, 053415 (2002).

[18] R. Moshammer et al., Phys. Rev. Lett. 91, 113002 (2003).

[19] J. Ullrich et al., Rep. Prog. Phys. 66, 1463 (2003).

[20] T. Jahnke et al., J. Electron. Spectrosc. Relat. Phenom. 141, 229 (2004).

[21] G. L. Yudin and M. Yu. Ivanov, Phys. Rev. A 64, 013409 (2001).

[22] A. M. Perelomov, V. S. Popov, and M. V. Terent'ev, Zh. Éksp. Teor. Fiz. 50, 1393 (1966) [Sov. Phys. JETP 23, 924 (1966)].

[23] T. Brabec, M. Y. Ivanov, and P. B. Corkum, Phys. Rev. A 54, R2551 (1996).

[24] B. Hu, J. Liu, and S. G. Chen, Phys. Lett. A 236, 533 (1997).

[25] J. Chen, J. Liu, and S. G. Chen, Phys. Rev. A 61, 033402 (2000).

[26] P. Salières, B. Carrè, L. Le Dèroff et al., Science 292, 902 (2001).

[27] M. Li et al., Phys. Rev. Lett. 112, 113002 (2014).

[28] X. Song et al., in preparation.

[29] F. H. M. Faisal, Nature Phys. 5, 319 (2009).

[30] The Coulomb-field-driven transverse backwardscattering may aslo happen at the third or even higher return times. However, the contribution of these higher return trajectories to the final momentum distribution is negligible according to our calculation.

[31] Ph. A. Korneev et al., Phys. Rev. Lett. 108, 223601 (2012).

[32] Note that the curved interference structure observed here is different from the structure corresponding to the outer prong of the fork as discussed in M. Möller et al., Phys. Rev. A 90, 023412 (2014) which has nothing to do with the interference between different electron trajectories and is not primarily caused by the Coulomb field.

[33] See Supplemental Material for details of the derivation of this expression.

[34] M. Kolesik and J. V. Moloney, Rep. Prog. Phys. 77, 016401 (2014). 
[35] M. Richter et al., Phys. Rev. Lett. 114, 143001 (2015).

[36] T. Marchenko, H. G. Muller, K. J. Schafer and M. J. J.

(2010). Varkking, J. Phys. B: At. Mol. Opt. Phys. 43, 185001 